\title{
THE (CO)HOMOLOGY OF GROUPS GIVEN BY PRESENTATIONS IN WHICH EACH DEFINING RELATOR INVOLVES AT MOST TWO TYPES OF GENERATORS
}

\author{
STEPHEN J. PRIDE
}

(Received 13 February 1990)

Communicated by H. Lausch

\begin{abstract}
Our set-up will consist of the following: (i) a graph with vertex set $V$ and edge set $E$; (ii) for each vertex $v \in V$ a non-trivial group $G_{v}$ given by a presentation $\left\langle\mathbf{x}_{v} ; \mathbf{r}_{v}\right\rangle$; (iii) for each edge $e=\{u, v\} \in E$ a group $G_{e}$ given by a presentation $\left\langle\mathbf{x}_{u}, \mathbf{x}_{v} ; \mathbf{r}_{e}\right\rangle$ where $\mathbf{r}_{e}$ consists of the elements of $r_{u} \cup r_{v}$ together with some further words on $x_{u} \cup \mathbf{x}_{v}$. We let $G=\langle\mathbf{x} ; \mathbf{r}\rangle$ where $\mathbf{x}=\bigcup_{v \in V} x_{v}, \mathbf{r}=\bigcup_{e \in E} \mathbf{r}_{e}$. Our aim is to try to describe the structure of $G$ in terms of the groups $G_{v}(v \in V), G_{e}(e \in E)$. Under suitable conditions the natural homomorphisms $G_{v} \rightarrow G(v \in V), G_{e} \rightarrow G(e \in E)$ are injective; and there is a short exact sequence

$$
0 \rightarrow \bigoplus_{v \in V}\left(\mathbf{Z} G \otimes_{G_{v}} I G_{v}\right)^{|\operatorname{Star}(v)|-1} \rightarrow \bigoplus_{e \in E}\left(\mathbf{Z} G \otimes_{G_{e}} I G_{e}\right) \rightarrow I G \rightarrow 0
$$

(where, for any group $H, I H$ is the augmentation ideal). Some (co)homological consequences of these results are derived.
\end{abstract}

1991 Mathematics subject classification (Amer. Math. Soc.): 20 F 05, 20 J 05.

\section{Introduction}

In [8], [9], [10] we considered groups given by presentations where the generating set is partitioned into non-empty disjoint subsets, and each defining relator involves symbols from exactly two of these subsets. Such a group $G$ can be expressed in the form

$$
\left\langle F_{v}(v \in V) ; \mathbf{t}\right\rangle,
$$

(C) 1992 Australian Mathematical Society $0263-6115 / 92 \$ A 2.00+0.00$ 
where each $F_{v}$ is a non-trivial free group, and $\mathbf{t}$ is a set of cyclically reduced elements of $*_{v \in V} F_{v}$, with each element of $t$ involving symbols from exactly two factors. Associated with $G$ we have a graph $\Gamma$ with vertex set $V$ and edge set

$E=\left\{\{u, v\}\right.$ : some element of $\mathbf{t}$ involves symbols from $F_{u}$ and $\left.F_{v}\right\}$.

For $e=\{u, v\} \in E$ we have a group $G_{e}=\left\langle F_{u}, F_{v} ; \mathbf{t}_{e}\right\rangle$, where $\mathbf{t}_{e}$ consists of all elements of $\mathrm{t}$ involving symbols from $F_{u}$ and $F_{v}$.

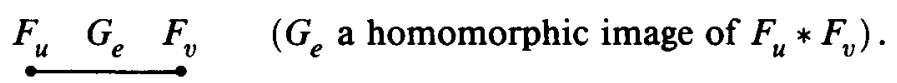

The idea is then to try to describe the structure of $G$ in terms of the groups $G_{e}$.

In this paper we replace the free groups $F_{v}$ by arbitrary non-trivial groups $G_{v}$. Our set-up will thus consist of the following.

(a) A graph $\Gamma$ with vertex set $V$ and edge set $E$. We assume for convenience that no vertex of $\Gamma$ is isolated.

(b) For each vertex $v \in V$ a group $G_{v}$ given by a presentation $\left\langle\mathbf{x}_{v} ; \mathbf{r}_{v}\right\rangle$, where the elements of $\mathbf{r}_{v}$ are cyclically reduced words on $\mathbf{x}_{v}$.

(c) For each edge $e=\{u, v\} \in E$, a group $G_{e}$ given by a presentation $\left\langle\mathbf{x}_{u}, \mathbf{x}_{v} ; \mathbf{r}_{e}\right\rangle$ where $\mathbf{r}_{e}$ consists of the elements of $\mathbf{r}_{u} \cup \mathbf{r}_{v}$, together with some cyclically reduced words each involving at least one $\mathbf{x}_{u}$-symbol and at least one $\mathbf{x}_{v}$-symbol.

We let

$$
G=\langle\mathbf{x} ; \mathbf{r}\rangle
$$

where

$$
\mathbf{x}=\bigcup_{v \in V} \mathbf{x}_{v}, \quad \mathbf{r}=\bigcup_{e \in E} \mathbf{r}_{e} .
$$

We can assume without loss of generality that $\mathbf{r}$ is slender, that is, if $R \in \mathbf{r}$ then no cyclic permutation of $R^{ \pm 1}$ except for $R$ itself belongs to $\mathrm{r}$.

EXAMPLE 1. If each $G_{v}$ is cyclic of order 2 and each $G_{e}$ is finite dihedral, then $G$ is a Coxeter group.

This example in fact motivated the work for this paper. In [11], R. Stöhr and I obtained a short exact sequence connecting the augmentation ideal of certain Coxeter groups (aspherical Coxeter groups) with the augmentation ideals of the cyclic and dihedral groups $G_{v}(v \in V), G_{e}(e \in E)$, and we used this to obtain results about (co)homology. The present paper arose from the realisation that the computations in [11] can be extended to the general set-up described above.

We will need to make two assumptions, which will be explained shortly. Later (see Theorem 3 ) we will give some fairly general sufficient conditions which ensure that our two assumptions hold. 
Assumption 1. The natural maps $G_{v} \rightarrow G(v \in V), G_{e} \rightarrow G(e \in E)$ are injective (and so we regard the $G_{v}$ and $G_{e}$ as subgroups of $G$ ).

To state the second assumption we need some definitions and notation (which will be used throughout the rest of the paper).

Write $G=F / N$, where $F$ is the free group on $\mathbf{x}$ and $N$ is the normal closure of $\mathbf{r}$ in $F$. We let $M$ denote the relation module for the given presentation of $G$. Thus $M$ is the left $\mathbb{Z} G$-module with underlying abelian group $N^{a b}=N / N^{\prime}$, and $G$-action

$$
W N \cdot U N^{\prime}=W U W^{-1} N^{\prime} \quad(W \in F, U \in N) .
$$

Similarly for $v \in V, e \in E$ we have relation modules $M_{v}, M_{e}$ (which are, respectively, left $\mathbb{Z} G_{v}-, \mathbb{Z} G_{\left.e^{-m o d u l e s}\right)}$ corresponding to the presentations of $G_{v}, G_{e}$. We also have the submodules $\bar{M}_{v}, \bar{M}_{e}$ of $M$ generated by $\left\{R N^{\prime}: R \in \mathbf{r}_{v}\right\},\left\{R N^{\prime}: R \in \mathbf{r}_{e}\right\}$ respectively.

For $e \in E$, let $P_{e}$ be the free left $\mathbb{Z} G$-module with basis $\left\{t_{R}^{e}: R \in \mathbf{r}_{e}\right\}$, and let $K_{e}$ be the kernel of the epimorphism

$$
P_{e} \rightarrow \bar{M}_{e}, t_{R}^{e} \mapsto R N^{\prime} \quad\left(R \in \mathbf{r}_{e}\right) .
$$

Let $P$ be the free left $\mathbb{Z} G$-module with basis $\left\{t_{R}: R \in \mathbf{r}\right\}$, and let $K$ be the kernel of the epimorphism

$$
P \rightarrow M, t_{R} \mapsto R N^{\prime} \quad(R \in \mathbf{r}) .
$$

Now we have an epimorphism

$$
\alpha: \bigoplus_{e \in E} P_{e} \rightarrow P
$$

given by

$$
t_{R}^{e} \mapsto t_{R} \quad\left(e \in E, R \in \mathbf{r}_{e}\right) .
$$

This obviously carries $\bigoplus_{e \in E} K_{e}$ into $K$.

Assumption 2. $\alpha$ carries $\bigoplus_{e \in E} K_{e}$ onto $K$.

We will have more to say about this assumption at the end of this introduction. The assumption is intimately connected with the structure of identity sequences over the presentation of $G$.

In the statements of Theorems 1 and 2, and the Corollaries, below, it will be supposed that Assumptions 1 and 2 hold.

THEOREM 1. There is a short exact sequence

$$
0 \rightarrow \bigoplus_{v \in V}\left(\mathbb{Z} G \otimes_{G_{v}} M_{v}\right)^{|S(v)|-1} \rightarrow \bigoplus_{e \in E} \mathbb{Z} G \otimes_{G_{e}} M_{e} \rightarrow M \rightarrow 0
$$


(Here $S(v)$ is the adjacency set of $v$, that is, the set of edges of $\Gamma$ incident with $v$.)

COROLlARY 1.1. There is a short exact sequence

$$
0 \rightarrow \bigoplus_{v \in V}\left(\mathbb{Z} G \otimes_{G_{v}} I G_{v}\right)^{|S(v)|-1} \rightarrow \bigoplus_{e \in E} \mathbb{Z} G \otimes_{G_{e}} I G_{e} \rightarrow I G \rightarrow 0 .
$$

(Here, for any group $H, I H$ is the augmentation ideal.)

Corollary 1.2. Let $A$ be any right $\mathbb{Z} G$-module, and let $B$ be any left $\mathbb{Z} G$-module.

(i) There is a long exact sequence

$$
\begin{aligned}
\cdots & \rightarrow H_{n+1}(G, A) \rightarrow \bigoplus_{v \in V} H_{n}\left(G_{v}, A\right)^{|S(v)|-1} \rightarrow \bigoplus_{e \in E} H_{n}\left(G_{e}, A\right) \\
& \rightarrow H_{n}(G, A) \rightarrow \cdots
\end{aligned}
$$

terminating in

$$
\cdots \rightarrow H_{2}(G, A) \rightarrow \bigoplus_{v \in V}\left(A \otimes_{G_{v}} I G_{v}\right)^{|S(v)|-1} \rightarrow \bigoplus_{e \in E} A \otimes_{G_{e}} I G_{e} \rightarrow A \otimes_{G} I G \rightarrow 0 .
$$

(ii) There is a long exact sequence

$$
\begin{aligned}
\cdots & \rightarrow H^{n}(G, B) \rightarrow \prod_{e \in E} H^{n}\left(G_{e}, B\right) \rightarrow \prod_{v \in V} H^{n}\left(G_{v}, B\right)^{|S(v)|-1} \\
& \rightarrow H^{n+1}(G, B) \rightarrow \cdots
\end{aligned}
$$

starting with

$$
\begin{aligned}
0 & \rightarrow \operatorname{Hom}_{\mathrm{z} G}(I G, B) \rightarrow \prod_{e \in E} \operatorname{Hom}_{\mathrm{z} G_{e}}\left(I G_{e}, B\right) \\
& \rightarrow \prod_{v \in V}\left(\operatorname{Hom}_{\mathbf{z} G_{v}}\left(I G_{v}, B\right)\right)^{|S(v)|-1} \rightarrow H^{2}(G, B) \rightarrow \cdots .
\end{aligned}
$$

REMARK. In the above Corollary, ( $)^{|S(v)|-1}$ is to be interpreted as direct sum in(i), and direct product in (ii).

CoRollary 1.3. Suppose there exists $n \geq 1$ such that $G_{v}$ has cohomological dimension less than or equal to $n$ for all $v \in V$. Then any finite subgroup of $G$ is contained in a conjugate of some subgroup $G_{e}(e \in E)$.

A proof of Theorem 1 will be given in Section 2 .

The proof of Corollary 1.1 is similar to arguments used in the proof of [11,Theorem 4]. (Details are left to the reader.) The proof of Corollary 
1.2 is obtained by considering the long exact Tor and Ext sequences (in the second and first variables respectively) derived from the short exact sequence in Corollary 1.1, and then using dimension shifting arguments and Shapiro's Lemma. The computations are similar to those in [11, Section 4], and are left to the reader. To prove Corollary 1.3 , observe that under the stated conditions we have

$$
H^{n+2}(G, B) \cong \prod_{e \in E} H^{n+2}\left(G_{e}, B\right)
$$

for all left $\mathbb{Z} G$-modules $B$. Now use Serre's Theorem [6].

Before stating the next theorem, we recall the definition of a combinatorially aspherical group (for further information see [2] or [3]).

Let a group $H$ be given by a presentation $\langle\mathbf{y} ; \mathbf{s}\rangle$, so that $H$ is (isomorphic to) $\Phi / \Sigma$, where $\Phi$ is the free group on $\mathbf{y}$ and $\Sigma$ is the normal closure of $\mathbf{s}$ in $\Phi$. For $S \in \mathbf{s}$ write $S=\dot{S}^{p(S)}$, where $\dot{S}$ is not a proper power and $p(S)$ is a positive integer. Let $X$ be the free left $\mathbb{Z} H$-module with basis $\left\{b_{S}: S \in \mathbf{s}\right\}$. We say that $H$ (or more precisely the given presentation of $H$ ) is combinatorially aspherical (CA) if the kernel of the epimorphism

$$
b_{S} \mapsto S \Sigma^{\prime} \quad(S \in \mathbf{s})
$$

from $X$ onto the relation module $\Sigma / \Sigma^{\prime}$, is generated by the elements $(1-\dot{S} \Sigma) b_{S}(S \in \mathbf{s})$.

Theorem 2. If each $G_{e}$ is $C A$ then $G$ is $C A$.

This theorem will be proved in Section 3 .

We now give conditions which guarantee that Assumptions 1 and 2 hold. Let $e=\{u, v\}$ be an edge of $\Gamma$. We will say that $G_{e}$ (or more precisely the given presentation of $G_{e}$ ) has property- $W_{k}$ if no non-trivial element of $G_{u} * G_{v}$ of free product length less than or equal to $2 k$ lies in the kernel of the natural epimorphism $G_{u} * G_{v} \rightarrow G_{e}$.

Example 2 (E. Fennessey). For $e=\{u, v\}$, let $D_{e}$ denote the cartesian subgroup of $G_{u} * G_{v}$ (that is, $D_{e}$ is the kernel of the natural epimorphism $\left.G_{u} * G_{v} \rightarrow G_{u} \times G_{v}\right)$. Let $\hat{\mathbf{r}}_{e}=\mathbf{r}_{e}-\left(\mathbf{r}_{u} \cup \mathbf{r}_{v}\right)$, where we regard the elements of $\hat{\mathbf{r}}_{e}$ as belonging to $G_{u} * G_{v}$. Then $G_{e}$ has property- $W_{1}$ if $\hat{\mathbf{r}}_{e} \subseteq D_{e}$, and $G_{e}$ has property- $W_{2}$ if $\hat{\mathbf{r}}_{e} \subseteq D_{e}^{p(e)} D_{e}^{\prime}$ for some prime $p(e)$.

EXAMPLe 3 (see M. Edjvet [4, Example 4.1]). For $v \in V$, let $\mathbf{x}_{v}$ consist of a single element (which we take to be $v$ itself). Let $\varphi$ be a mapping from $V \cup E$ into $\{2,3,4, \ldots\}$, and let

$$
G_{e}=\left\langle u, v ; u^{\varphi(u)}, v^{\varphi(v)},(u v)^{\varphi(e)}\right\rangle(e=\{u, v\} \in E) .
$$

Then $G_{e}$ always has property- $W_{1}$, and has property- $W_{2}$ if $\varphi(e)>2$. 
THEOREM 3. Assumptions 1 and 2 hold if either of the following conditions is satisfied.

(I) Each $G_{e}$ has property- $W_{2}$.

(II) $\Gamma$ is triangle-free and each $G_{e}$ has property- $W_{1}$.

Remarks. (1) A proof of the theorem will be given in Section 4. Another proof (based on extending results on van Kampen and spherical diagrams over free groups obtained in [8], [9], [10], to analogous results for diagrams over free products) has been given by E. Fennessey [5].

(2) Note that Theorem 3 applies to many Coxeter groups (take $\varphi(v)=2$ for all $v \in V$ in Example 3). We remark that, for Coxeter groups, Assumption 1 always holds [12, p. 245]. To guarantee that Assumption 2 holds we can get by with a weaker condition than (I)/(II), namely the asphericity condition [11] referred to earlier.

(3) We can, in fact, formulate an asphericity condition for our general set-up, as follows. For $e=\{u, v\} \in E$, let $m_{e}$ be the length of the shortest non-trivial element of $\operatorname{Ker}\left(G_{u} * G_{v} \rightarrow G_{e}\right)$. (Note that either $m_{e}=1$ (which means that one of the natural homomorphisms $G_{u} \rightarrow G_{e}, G_{v} \rightarrow G_{e}$ is not injective), or $m_{e}$ is even.) The asphericity condition would require that:

(i) $m_{e} \geq 4$ for all $e$ (that is, each $G_{e}$ has property- $W_{1}$ );

(ii) for any triangle $\Gamma$ (with edges $e_{1}, e_{2}, e_{2}$, say)

$$
\frac{1}{m_{e_{1}}}+\frac{1}{m_{e_{2}}}+\frac{1}{m_{e_{3}}} \leq \frac{1}{2}
$$

It seems plausible that an analogue of Theorem 3 could be obtained under the weaker asphericity condition.

To complete this introduction we now discuss Assumption 2 in more detail. We first briefly review some basic ideas concerning identity sequences (for further information, see [2], especially Sections 2, 3).

Let $\mathbf{w}$ be the set of words on $\mathbf{x}$ (reduced or not). For $\mathbf{s} \subseteq \mathbf{r}$, let $\mathbf{s}^{\mathbf{w}}$ denote the subset of $w$ consisting of all words of the form

$$
W S^{\varepsilon} W^{-1} \quad(W \in \mathbf{w}, S \in \mathbf{s}, \varepsilon= \pm 1) .
$$

If $C=W R^{\varepsilon} W^{-1}$ is an element of $\mathbf{r}^{\mathbf{W}}$ then we define eval $(C)$ to be the element $\varepsilon W N t_{R}$ of the free module $P$. If $\sigma=\left(C_{1}, C_{2}, \ldots, C_{n}\right)$ is a finite sequence of elements of $\mathbf{r}^{\mathbf{w}}$ then we define $\operatorname{eval}(\sigma)$ to be $\sum_{i=1}^{n} \operatorname{eval}\left(C_{i}\right)$. If $V \in \mathrm{w}$ then $\sigma^{V}$ is the sequence $\left(V C_{1} V^{-1}, V C_{2} V^{-1}, \ldots, V C_{n} V^{-1}\right)$.

A finite sequence $\left(C_{1}, C_{2}, \ldots, C_{n}\right)$ of elements $C_{i}=W_{i} R_{i}^{\varepsilon_{i}} W_{i}^{-1}$ of elements of $\mathbf{r}^{\mathbf{m}}$ is called an identity sequence if $C_{1} C_{2} \ldots C_{n}$ is freely equal to 1 . We define operations on such sequences as follows.

(1) Replace $W_{i}$ by a word freely equal to it for $i=1, \ldots, n$. 
(2) Delete two successive terms $C_{j}, C_{j+1}$ if $C_{j+1}$ is identically equal to $C_{j}^{-1}$.

(3) Replace two successive terms $C_{j}, C_{j+1}$ by either $C_{j+1}, C_{j+1}^{-1} C_{j} C_{j+1}$ or $C_{j} C_{j+1} C_{j}^{-1}, C_{j}$. Two identity sequences are said to be Peiffer equivalent if one can be obtained from the other by a finite number of operations (1), $(2),(2)^{-1}$ (the operation inverse to (2)), and (3). The Peiffer equivalence class of a sequence $\sigma$ will be denoted by $\langle\sigma\rangle$. The set of Peiffer equivalence classes is a left $\mathbb{Z} G$-module with addition defined by

$$
\langle\sigma\rangle+\langle\tau\rangle=\langle\sigma \tau\rangle
$$

and $G$-action defined by

$$
W N \cdot\langle\sigma\rangle=\left\langle\sigma^{W}\right\rangle .
$$

This $\mathbb{Z} G$-module is isomorphic to $K$ under the mapping

$$
\langle\sigma\rangle \mapsto \operatorname{eval}(\sigma) .
$$

Consider the following assumption.

Assumption $2^{\prime}$. Every identity sequence $\sigma$ is Peiffer equivalent to a product $\sigma_{1} \sigma_{2} \ldots \sigma_{k}$ of identity sequences $\sigma_{i}(1 \leq i \leq k)$, where for $i=1, \ldots, k$ there is an edge $e_{i}$ of $\Gamma$ such that all terms of $\sigma_{i}$ belong to $\mathbf{r}_{i}^{\mathrm{w}}$.

It is easy to see that Assumption $2^{\prime}$ implies Assumption 2. Moreover, one can show that if Assumption 1 holds then Assumption 2 implies Assumption $2^{\prime}$ (see the second remark at the end of Section 3). Thus Assumptions 1 and 2 are equivalent to Assumptions 1 and $2^{\prime}$.

\section{Proof of Theorem 1}

We will need the following results.

Lemma 1. The submodules $\bar{M}_{v}(v \in V)$ generate their direct sum in $M$.

Proof. Let $Q$ be the free left $\mathbb{Z} G$-module with basis $\left\{b_{x}: x \in \mathbf{x}\right\}$. There is a standard short exact sequence

$$
0 \rightarrow M \stackrel{\mu}{\longrightarrow} Q \rightarrow I G \rightarrow 0
$$

where the embedding $\mu$ is given by

$$
W N^{\prime} \mapsto \Sigma_{x \in \mathbf{x}} \pi \frac{\partial W}{\partial x} b_{x} \quad(W \in F),
$$


with $\partial / \partial x$ Fox differentiation, and $\pi$ the natural projection $\mathbb{Z F} \rightarrow \mathbb{Z} G[1$, p. 43]. The image of $\bar{M}_{v}$ under $\mu$ lies in the submodule of $Q$ generated by $\left\{b_{x}: x \in \mathbf{x}_{v}\right\}$. $M_{v}$.

Lemma 2. For $e \in E, v \in V$ we have $\bar{M}_{e} \cong \mathbb{Z} G \otimes_{G_{e}} M_{e}, \bar{M}_{v} \cong \mathbb{Z} G \otimes_{G_{v}}$

Proof. Let $e=\{u, v\} \in E$, and let $Q_{e}$ be the free left $\mathbb{Z} G_{e}$-module with basis $\left\{b_{x}^{e}: x \in \mathbf{x}_{u} \cup \mathbf{x}_{v}\right\}$. Then we have a short exact sequence

$$
0 \rightarrow M_{e} \stackrel{\mu_{e}}{\longrightarrow} Q_{e} \rightarrow I G_{e} \rightarrow 0,
$$

analogous to (2.1). Tensoring by the free right $\mathbb{Z} G_{e}$-module $\mathbb{Z} G$ we get the exact sequence

$$
0 \rightarrow \mathbb{Z} G \otimes_{G_{e}} M_{e} \stackrel{1 \otimes \mu_{e}}{\longrightarrow} \mathbb{Z} G \otimes_{G_{e}} Q_{e} \rightarrow \mathbb{Z} G \otimes_{G_{e}} I G_{e} \rightarrow 0 .
$$

Now $\mathbb{Z} G \otimes_{G_{e}} Q_{e}$ is isomorphic to the submodule of $Q$ generated by $\left\{b_{x}: x \in\right.$ $\left.\mathbf{x}_{u} \cup \mathbf{x}_{v}\right\}$, under the mapping $1 \otimes b_{x}^{e} \mapsto b_{x}$. The image of $\left(1 \otimes \mu_{e}\right)\left(\mathbb{Z} G \otimes_{G_{e}} M_{e}\right)$ under this isomorphism is precisely $\mu\left(\bar{M}_{e}\right)$. Thus $\bar{M}_{e} \cong \mathbb{Z} G \otimes_{G_{e}} M_{e}$, as required.

The other result is similar.

Assumption 2 implies that the kernel of the induced homomorphism

$$
\alpha^{*}: \frac{\bigoplus P_{e}}{\bigoplus K_{e}} \rightarrow \frac{P}{K}
$$

consists of the cosets $\bmod \oplus K_{e}$ of the elements of $\operatorname{Ker} \alpha$. We note that $\operatorname{Ker} \alpha$ is (freely) generated by the elements

$$
t_{R}^{e}-t_{R}^{e(v)} \quad\left(v \in V, e \in S^{\prime}(v), R \in \mathbf{r}_{v}\right) .
$$

(Here $e(v)$ is some fixed element of $S(v)$, and $S^{\prime}(v)=S(v)-\{e(v)\}$.)

Now $\oplus P_{e} / \oplus K_{e} \cong \oplus \bar{M}_{e}$. Let $f=\{u, v\}$ be an edge of $\Gamma$ and let $\bar{M}_{u}^{f}$ be the module $\bar{M}_{u}$ regarded as a submodule of the factor $\bar{M}_{f}$ of $\oplus \bar{M}_{e}$, and similarly for $v$. Then $\bar{M}_{u}^{f} \cap \bar{M}_{v}^{f}=0$, by Lemma 1 , so we get the submodule

$$
\begin{aligned}
L & =\bigoplus_{\{u, v\} \in E}\left(\bar{M}_{u}^{\{u, v\}} \oplus \bar{M}_{v}^{\{u, v\}}\right) \\
& =\bigoplus_{v \in V} \bigoplus_{e \in S(v)} \vec{M}_{v}^{e}
\end{aligned}
$$


of $\oplus \bar{M}_{e}$. The submodule of $L$ corresponding to $\operatorname{Ker} \alpha^{*}$ is generated by the elements

$$
m^{e}-m^{e(v)} \quad\left(v \in V, \quad e \in S^{\prime}(v), \quad m \in \bar{M}_{v}\right) .
$$

(Here $m^{e}$ is the element $m$ of $\bar{M}_{v}$ thought of as an element of the factor $\bar{M}_{e}$ of $\oplus \bar{M}_{e}$, and similarly for $m^{e(v)}$.) Now there is an automorphism of $L$ defined by

$$
\begin{aligned}
m^{e} \mapsto m^{e}-m^{e(v)} & & \left(v \in V, e \in S^{\prime}(v), m \in \bar{M}_{v}\right), \\
m^{e(v)} \mapsto m^{e(v)} & & \left(v \in V, m \in \bar{M}_{v}\right) .
\end{aligned}
$$

The image of $\bigoplus_{v \in V} \bigoplus_{e \in S^{\prime}(v)} \bar{M}_{v}^{e}$ under this automorphism is $\operatorname{Ker} \alpha^{*}$. Consequently we have a short exact sequence

$$
0 \rightarrow \bigoplus_{v \in V} \bigoplus_{e \in S^{\prime}(v)} \bar{M}_{v}^{e} \rightarrow \bigoplus_{e \in E} \bar{M}_{e} \rightarrow M \rightarrow 0
$$

Now Theorem 1 follows using Lemma 2.

\section{Proof of Theorem 2}

For $e=\{u, v\}$ an edge of $\Gamma$, write $G_{e}=F_{e} / N_{e}$, where $F_{e}$ is the free group on $\mathbf{x}_{u} \cup \mathbf{x}_{v}$, and $N_{e}$ is the normal closure of $\mathbf{r}_{e}$ in $F_{e}$. The relation module $M_{e}$ is then $N_{e} / N_{e}^{\prime}$. Let $A_{e}$ be the free left $\mathbb{Z} G$-module with basis $\left\{a_{R}^{e}: R \in \mathbf{r}_{e}\right\}$. By assumption the kernel $B_{e}$ of the epimorphism

$$
A_{e} \rightarrow M_{e}, \quad a_{R}^{e} \mapsto R N_{e}^{\prime} \quad\left(R \in \mathbf{r}_{e}\right)
$$

is generated by the elements $\left(1-\dot{R} N_{e}\right) a_{R}^{e}\left(R \in \mathbf{r}_{e}\right)$. Tensoring the exact sequence

$$
0 \rightarrow B_{e} \rightarrow A_{e} \rightarrow M_{e} \rightarrow 0
$$

by the free right $\mathbb{Z} G_{e}$-module $\mathbb{Z} G$ gives the exact sequence

$$
0 \rightarrow \mathbb{Z} G \otimes_{G_{e}} B_{e} \rightarrow \mathbb{Z} G \otimes_{G_{e}} A_{e} \rightarrow \mathbb{Z} G \otimes_{G_{e}} M_{e} \rightarrow 0
$$

Now we have isomorphisms

$$
\begin{array}{r}
\mathbb{Z} G \otimes_{G_{e}} A_{e} \stackrel{1 \otimes a_{R}^{e} \mapsto t_{R}^{e}}{\longrightarrow} P_{e}, \\
\mathbb{Z} G \otimes_{G_{e}} M_{e} \stackrel{1 \otimes R N_{e}^{\prime} \mapsto R N^{\prime}}{\longrightarrow} \bar{M}_{e}
\end{array}
$$

(the latter by Lemma 2). Thus we obtain an isomorphism

$$
\mathbb{Z} G \otimes_{G_{e}} B_{e} \rightarrow K_{e}
$$


carrying the generating set

$$
\left\{1 \otimes\left(1-\dot{R} N_{e}\right) a_{R}^{e}: R \in \mathbf{r}_{e}\right\}
$$

of $\mathbb{Z} G \otimes_{G_{e}} B_{e}$ to

$$
\left\{(1-\dot{R} N) t_{R}^{e}: R \in \mathbf{r}_{e}\right\} .
$$

Since $\alpha: \bigoplus K_{e} \rightarrow K$ is onto, we deduce that $K$ is generated by the elements

$$
(1-\dot{R} N) t_{R} \quad\left(R \in \mathbf{r}_{e}, e \in E\right),
$$

as required.

REMARKs. (1) We can obviously prove the following result by similar arguments.

Suppose Assumptions 1 and 2 hold. For $e \in E$, let $X_{e}$ be a set of generators for the $\mathbb{Z} G$-module $B_{e}$. Then $1 \otimes_{G_{e}} X_{e}=\left\{1 \otimes x: x \in X_{e}\right\}$ is a set of generators for the $\mathbb{Z} G$-module $\mathbb{Z} G \otimes_{G_{e}} B_{e}$, and so the image of $\bigcup_{e \in E} 1 \otimes_{G_{e}} X_{e}$ under the composition

$$
\bigoplus_{e \in E} \mathbb{Z} G \otimes_{G_{e}} B_{e} \stackrel{(3.1)}{\longrightarrow} \bigoplus_{e \in E} K_{e} \stackrel{\alpha}{\rightarrow} K
$$

is a generating set of $K$.

(2) In particular, for $e=\{u, v\} \in E$, let $\mathbf{w}_{e}$ be the set of words on $\mathbf{x}_{u} \cup \mathbf{x}_{v}$. Let $\Sigma_{e}$ be the set of identity sequences of elements of $\mathbf{r}_{e}^{w_{e}}$. If $\tau=\left(W_{1} R_{1}^{\varepsilon_{1}} W_{1}^{-1}, \ldots, W_{n} R_{n}^{\varepsilon_{n}} W_{n}^{-1}\right)$ is an element of $\Sigma_{e}$, let eval $e_{e}(\tau)$ denote the element $\varepsilon_{1} W_{1} N_{e} a_{R_{1}}^{e}+\cdots+\varepsilon_{n} W_{n} N_{e} a_{R_{n}}^{e}$ of $A_{e}$. Then $B_{e}=\left\{\operatorname{eval}_{e}(\tau)\right.$ : $\left.\tau \in \Sigma_{e}\right\}$. The image of $\left\{1 \otimes \operatorname{eval}_{e}(\tau):{ }^{n} \tau \in \Sigma_{e}, e \in E\right\}$ under (3.2) is $\left\{\operatorname{eval}(\tau): \tau \in \Sigma_{e}, e \in E\right\}$. From this we deduce that if $\sigma$ is an identity sequence of elements of $r^{w}$ then

$$
\operatorname{eval}(\sigma)=V_{1} N \operatorname{eval}\left(\tau_{1}\right)+\cdots+V_{m} N \operatorname{eval}\left(\tau_{m}\right)
$$

for certain elements $V_{i} N$ of $G$ and elements $\tau_{i}$ of $\bigcup_{e \in E} \Sigma_{e}$. Thus

$$
\langle\sigma\rangle=\left\langle\tau_{1}^{V_{1}} \cdots \tau_{m}^{V_{m}}\right\rangle
$$

\section{Proof of Theorem 3}

For $e=\{u, v\} \in E$, we will write $\hat{\mathbf{r}}_{e}$ for the set $\mathbf{r}_{e}-\left(\mathbf{r}_{u} \cup \mathbf{r}_{v}\right)$, and we will denote the union of all the $\hat{\mathbf{r}}_{e}$ 's by $\hat{\mathbf{r}}$. The free product $G_{u} * G_{v}$ will be denoted by $\widetilde{G}_{e}$.

We will be interested in diagrams over the presentation $\langle\mathbf{x} ; \mathbf{r}\rangle$ of $G$. (For the general theory of diagrams over presentations, see [7, Chapter V].) Our 
main concern will be with what we will call $\mathbf{r}$-discs and r-spheres. An r-disc (respectively r-sphere) is a finitely tesselated disc (respectively, sphere) whose oriented edges are labelled by elements of $\mathbf{x} \cup \mathbf{x}^{-1}$, and where each region has a boundary path labelled by an element of $\mathbf{r}$.

Let $\mathbf{M}$ be an r-disc or r-sphere. We let $E(\mathbf{M})$ denote the set of regions of $\mathbf{M}$ which are labelled by elements of $\hat{\mathbf{r}}$. If $\Delta \in E(\mathbf{M})$, and if the label on $\Delta$ belongs to $\hat{\mathbf{r}}_{e}$ then we define $e(\Delta)$ to be $e$. For any path $\alpha$ in $\mathbf{M}$, we let $t p(\alpha)$ denote the set of all $v \in V$ for which some element of $\mathbf{x}_{v}$ occurs in the label of $\alpha$.

Suppose $E(\mathbf{M}) \neq \varnothing$. Choose some $\Delta \in E(\mathbf{M})$, and consider the collection of all regions $\Delta^{\prime}$ of $\mathbf{M}$ for which the following holds: there exist regions $\Delta=$ $\Delta_{0}, \Delta_{1}, \ldots, \Delta_{n}=\Delta^{\prime}$ with $t p\left(\partial \Delta_{i}\right) \subseteq e(\Delta) \quad(i=1, \ldots, n)$, and where $\Delta_{i}$, $\Delta_{i+1}$ have an edge in common for $i=0, \ldots, n-1$. We call this collection, and also the subdiagram $\mathbf{F}$ of $\mathbf{M}$ which it determines, a federation. We define $e(\mathbf{F})$ to be $e(\Delta)$.

Let $F_{1}$ be a federation of $M$. If $F_{1} \neq M$, then construct a federation $\mathbf{F}_{2}$ of $\mathbf{M}-\mathbf{F}_{1}$. (The reader at this point may object, since we have only defined the concept of federation for r-discs and r-spheres. However, it is clear that we can define the concept of federation for subdiagrams of r-discs.) If $\mathbf{F}_{2} \neq \mathbf{M}-\mathbf{F}_{1}$, then choose a federation $\mathbf{F}_{3}$ of $\mathbf{M}-\left(\mathbf{F}_{1} \cup \mathbf{F}_{2}\right)$, and so on. Eventually, we will get a collection $\mathbf{F}_{1}, \ldots, \mathbf{F}_{n}$ of subdiagrams of $\mathbf{M}$ which cover $\mathbf{M}$, with $\mathbf{F}_{i+1}$ a federation of $\mathbf{M}-\bigcup_{j=1}^{i} \mathbf{F}_{j}$. We call $\left\{\mathbf{F}_{i}\right\}_{i=1}^{n}$ a federal subdivision of $\mathbf{M}$.

Lemma 3. Let $\mathbf{D}$ be an $\mathbf{r}$-disc having a federal subdivision $\left\{\mathbf{F}_{i}\right\}_{i=1}^{n}$ with the following properties: (i) each $\mathbf{F}_{i}$ is simply connected; (ii) the label on $\partial \mathbf{F}_{i}$ defines a non-trivial element of the kernel of the natural epimorphism $\tilde{G}_{e\left(\mathbf{F}_{i}\right)} \rightarrow G_{e\left(\mathbf{F}_{i}\right)}(i=1, \ldots, n)$. Then there exists some $\mathbf{F}_{j}$ such that $\mathbf{F}_{j} \cap \partial \mathbf{D}$ contains at least one edge, and $e\left(\mathbf{F}_{j}\right) \subseteq t p(\partial \mathbf{D})$.

This is a special case of the proof of the main lemma of Edjvet's paper [4]. (We remark that, strictly speaking, Edjvet's result only applies when each $\mathbf{x}_{v}$ is a singleton. However, the extension to the more general situation where the $\mathbf{x}_{v}$ 's may have more than one element is easy.)

The proof that Assumption 1 holds now follows from Lemma 3 using arguments similar to those in the proof of Theorem 1 of [6] (see also the proof of Theorem 4 of [6]).

LEMma 4. Let $\mathbf{S}$ be an $\mathbf{r}$-sphere having a federal subdivision $\left\{\mathbf{F}_{i}\right\}_{i=1}^{n}$ with $n>1$. Then there is a simply connected federation $\mathbf{F}$ whose boundary label 
defines the identity of $\operatorname{Ker}\left(\tilde{G}_{e(\mathbf{F})} \rightarrow G_{e(\mathbf{F})}\right)$. Moreover, for any given vertex of $\mathbf{S}, \mathbf{F}$ can be chosen so that that vertex does not lie in the interior of $\mathbf{F}$.

Proof. By arguing as on p. 573 of [9], we can find some federation $\mathbf{F}_{p}$ and a component $\mathbf{C}$ of $\mathbf{S}-\mathbf{F}_{p}$ with all $\mathbf{F}_{j} \subseteq \mathbf{C}$ simply connected. Suppose that for each $\mathbf{F}_{j} \subseteq \mathbf{C}$ the boundary label of $\mathbf{F}_{j}$ defines a non-trivial element of $\operatorname{Ker}\left(\widetilde{G}_{e\left(\mathbf{F}_{j}\right)} \rightarrow G_{e\left(\mathbf{F}_{j}\right)}\right)$ (the boundary label must define an element of this kernel). Then by Lemma 1 (applied to $\mathbf{C}$ ), there exists $\mathbf{F}_{q} \subseteq \mathbf{C}$ with $\mathbf{F}_{q} \cap \partial \mathbf{C}$ containing at least one edge, and $e\left(\mathbf{F}_{q}\right) \subseteq t p(\partial C)$. Now $t p(\partial \mathbf{C}) \subseteq e\left(\mathbf{F}_{p}\right)$, so $e\left(\mathbf{F}_{q}\right)=e\left(\mathbf{F}_{p}\right)$. Since $\mathbf{F}_{q}$ and $\mathbf{F}_{p}$ have an edge in common, this contradicts the fact that $\mathbf{F}_{p}$ and $\mathbf{F}_{q}$ are federations (they should have been incorporated into one larger federation!).

The last statement of the lemma follows as on the bottom of p. 104 of [10].

We will now show that Assumption $2^{\prime}$ holds.

If $\sigma$ is an identity sequence then we define the $\operatorname{rank} \operatorname{rk}(\sigma)$ of $\sigma$ to be the ordered pair $\left(n_{1}, n_{2}\right)$, where $n_{1}$ is the number of terms belonging to $\hat{\mathbf{r}}^{\|}$, and $n_{2}$ is the number of terms belonging to $\mathbf{r}^{n}-\hat{\mathbf{r}}^{\boldsymbol{m}}$. The proof is by induction on rank (where ordered pairs are given the lexicographical ordering).

If $\operatorname{rk}(\sigma)=(0,0)$ there is nothing to prove.

Suppose $\operatorname{rk}(\sigma)>(0,0)$. We are going to use the theory of spherical pictures, which is a standard geometric tool for detailing with identity sequences (see [2], [10], for the general theory).

Construct a spherical picture $\mathbf{P}$, having a spray whose associated identity sequence is $\sigma$ (see $[10, \mathbf{p} .103])$. Let $\mathbf{P}^{\prime}$ be a spherical subpicture of $\mathbf{P}$ containing at least one disc of $\mathbf{P}$, and which is minimal with respect to this property.

Assume first that some disc of $\mathbf{P}^{\prime}$ is labelled by an element of $\hat{\mathbf{r}}$. Then dualising $\mathbf{P}^{\prime}$ to obtain an $\mathbf{r}$-sphere, and arguing as in [10, pp. 103-105] (making use of Lemma 4 above), we can find a simple closed transverse path $\delta$ in $\mathbf{P}$ with the following properties.

(4.1) $\delta$ does not intersect any disc of $\mathbf{P}$, and does not intersect the boundary of $\mathbf{P}$.

(4.2) There is at least one disc of $\mathbf{P}$ inside $\delta$.

(4.3) There is some $e=\{u, v\} \in E$ such that all discs inside $\delta$ are labelled by elements of $\mathbf{r}_{e}$.

(4.4) At least one disc inside $\delta$ is labelled by an element of $\hat{\mathbf{r}}_{e}$.

(4.5) The label $U$ on $\delta$ defines the identity of $\widetilde{G}_{e}=G_{u} * G_{v}$.

Let $k, l$ be the number of discs inside and outside $\delta$ respectively. Then 
we can construct a spray for $\mathbf{P}$ where the first $k$ paths are connected to the discs inside $\delta$ and the remaining $l$ paths are connected to the discs outside $\delta$, and where the identity sequence $\sigma^{\prime}=\left(C_{1}, C_{2}, \ldots, C_{k}, C_{k+1}, \ldots, C_{k+l}\right)$ associated with the spray is such that $C_{1} C_{2} \ldots C_{k}$ is freely equal to a conjugate $Z U Z^{-1}$ of $U$ (see [10, pp. 105-106]). It is important to note that $\sigma$ and $\sigma^{\prime}$ are Peiffer equivalent. For since $\sigma$ and $\sigma^{\prime}$ both arise from sprays, $\sigma^{-1} \sigma^{\prime}$ has the primary identity property as defined in [2, p. 177] (see [10], especially statement (1.3) on p. 102, in this regard). Hence [2, Proposition 16(ii)] applies.

Now since $U$ defines the identity of $G_{u} * G_{v}$ there is a sequence $\left(D_{1}, \ldots, D_{m}\right)$ of elements of $\left(\mathbf{r}_{u} \cup \mathbf{r}_{v}\right)^{\prime \prime}$ such that $D_{1} D_{2} \ldots D_{m}$ is freely equal to $Z U Z^{-1}$. Let

$$
\begin{aligned}
\sigma_{1} & =\left(C_{1}, \ldots, C_{k}, D_{m}^{-1}, \ldots, D_{1}^{-1}\right) \\
\tau & =\left(D_{1}, \ldots, D_{m}, C_{k+1}, \ldots, C_{k+l}\right) .
\end{aligned}
$$

Then $\sigma_{1} \tau$ is Peiffer equivalent to $\sigma^{\prime}$ (and hence to $\sigma$ ). Also, $\sigma_{1}$ is an identity sequence all of whose terms lie in $\mathbf{r}_{e}^{\mathbf{m}}$, and $\tau$ is an identity sequence with $\operatorname{rk}(\tau)<\operatorname{rk}(\sigma)$. Now apply the inductive hypothesis to $\tau$.

Next suppose that no disc of $\mathbf{P}^{\prime}$ is labelled by an element of $\hat{\mathbf{r}}$. Then all discs of $\mathbf{P}^{\prime}$ are labelled by elements of $\mathbf{r}_{v}$ for some $v \in V$. By taking $\delta$ to be a suitable path encircling $\mathbf{P}^{\prime}$, and by choosing an edge $e$ incident with $v$, we have that (4.1)-(4.3) hold, and instead of (4.4) and (4.5) we have that the label $U$ on $\delta$ is the empty word. It is now an easy matter to adapt the arguments of the previous two paragraphs to obtain the required result.

\section{Acknowledgement}

I thank Eric Fennessey for his useful comments.

\section{References}

[1] K. S. Brown, Cohomology of groups (Springer, 1982).

[2] R. Brown and J. Huebschmann, 'Identities among relations', Low Dimensional Topology, London Math. Soc. Lecture Notes 48 edited by R. Brown and T. L. Thickston (Cambridge University Press, 1982).

[3] I. M. Chiswell, D. J. Collins and J. Huebschmann, 'Aspherical group presentations', Math. Z. 178 (1981), 1-36.

[4] M. Edjvet, 'On a certain class of group presentations', Math. Proc. Cambridge Philos. Soc. 105 (1989), 25-35. 
[5] E. J. Fennessey, (Ph.D. Thesis, University of Glasgow, 1989).

[6] J. Huebschmann, 'Cohomology theory of aspherical groups and of small cancellation groups', J. Pure Appl. Algebra 14 (1979), 137-143.

[7] R. C. Lyndon and P. E. Schupp, Combinatorial Group Theory (Springer, 1977).

[8] S. J. Pride, 'Groups with presentations in which each defining relator involves exactly two generators', J. London Math. Soc. (2) 36 (1987), 245-256.

[9] S. J. Pride, 'The diagrammatic asphericity of groups given by presentations in which each defining relator involves exactly two types of generators', Arch. Math. 50 (1988), 570-574.

[10] S. J. Pride and R. Stöhr, 'Relation modules of groups with presentations in which each relator involves exactly two types of generator', J. London Math. Soc. (2) 38 (1988), 99-111.

[11] S. J. Pride and R. Stöhr, 'The (co)homology of aspherical Coxeter groups', J. London Math. Soc. (2) 42 (1990), 49-63.

[12] M. Suzuki, Group Theory I (Grundlehren. Math. Wiss. 247, Springer, 1982).

University Gardens

University of Glasgow

Glasgow G12 8QW

Scotland UK 